\title{
CHOICE OF THREE DIFFERENTS INTRAMEDULLARY NAILS IN THE TREATMENT OF TROCHANTERIC FRACTURES: OUTCOME, ANALYSIS AND CONSIDERATION IN \\ MIDTERM
}

\section{AUTHORS}

Persiani Pietro MD PhD 1 Grasso Raffaele MD 1 Gurzi Michele MD 1 Formica Alessandro MD 2 Graci Iole MD 1 De Cristo Claudia MD 1 Villani Ciro MD Chief 1

1: Department of Anatomical, Histological, Forensic Medicine and Orthopedic Science, University of Rome "Sapienza," Rome, Italy.

2: Orthopaedic Department, San Giovanni-Addolorata Hospital, Rome, Italy.

Author for correspondence: Raffaele Grasso MD, phone: +39 3392450355, e-mail: dr.raffaelegrasso@gmail.com.

KEYWORDS: Intramedullary nail; Proximal femoral fracture; Gamma nail; Intertrochanteric fractures; ZNN nail; Affixus nail. 


\section{ABSTRACT}

The purpose of this study is to compare the results obtained using three different systems of osteosynthesis, developed for the surgical treatment of fractures of the trochanteric region of the femur, based on the principle intramedullary nailing: the Gamma nail, the Affixus nail and the ZNN nail. This is a retrospective study: 72 trochanteric fractures treated with the Gamma nail, 68 treated with the Affixus nail and 69 treated with the ZNN nail, between the years 2012 and 2014, with the prerequisite of a minimum follow-up of 18 months. The fractures were classified according to the AO system; the most commonly reported subtype was the A2 fracture. Clinical and radiographic examinations were performed, both at hospital admission and post-operatively, at 1, 3, 6, 12 and 18 months. Of the 209 patients, 171 were women and 38 were men. The average age was 83.12 years old.

All three systems guaranteed an early mobilization and ambulation in most of the patients. There were no significant differences in the use of the three nails in terms of recovery of previous functional capacity, or in terms of the time required for the fracture to heal.

There were no advantages encountered with the use of one intramedullary nail over another and, in particular, when observing the complications and patient outcome, there were no statistically significant differences detected. 


\section{INTRODUCTION}

The fractures of the proximal femur are the most frequent traumatological occurrence after the age of 70 , with a female to male ratio of 2.5:1. Instead, atraumatic fractures, most commonly known as "fragility fractures", are due to higher bone fragility caused by osteoporosis $^{[1]}$. The elderly population is expected to increase from 600 million to 2 billion over the next 50 years, thus proximal femur fractures are predicted to double. In $20 \%$ of the patients an ambulatory disability remains, while $30-40 \%$ of them regain the autonomy to carry out daily activities [2-3]. The consequences of femoral fractures include an estimated mortality rate of about $5 \%$ in the acute phase and of $15-25 \%$ within one year. In fact, the annual mortality of elderly fractured patients exceeds that of many tumoral pathologies.

The treatment of choice is intramedullary nailing ${ }^{[4]}$, even though in the literature there is no consensus on which is the best intramedullary device for lateral femoral fractures ${ }^{[5-6-7]}$.

The purpose of this study is to assess the medium-term clinical and radiographic results obtained through the use of three different cephalo-medullary nails: straight nail with a cephalic screw (Gamma (C) Stryker Mahwah USA ${ }^{[8-9]}$ ), straight nail with cephalic screw and anti-rotational screw (Affixus (C) Depuy Orthopaedic Inc. Warsaw USA [10]) and anatomical nail with a cephalic screw (ZNN @ Zimmer Natural Nail Warsaw USA ${ }^{[11-12]}$ ).

\section{MATERIALS AND METHODS}

Out of a total of 711 patients treated from January $1^{\text {st }} 2012$ to January $1^{\text {st }} 2014$, for pertrochanteric fracture according to the 31-A.1, A.2 and A.3 AO classification, at the U.O.C. Orthopedics and Traumatology Department of Anatomical, Histological, Legal, Medical and Locomotive System Sciences, Rome "La Sapienza", a sample of 209 non-consecutive patients was selected. The patients included in the study were the ones for whom it was possible to have complete information from medical records and X-rays, and to whom it was possible to administer the Harris Hip Score ${ }^{[13-14]}$ and SF12 ${ }^{[15]}$ questionnaires.

The criteria of exclusion were: the presence of pathological fractures, poly-traumatized patients, patients with previous lateral femoral fracture, patients with a pre-fracture impairment of their ambulatory ability, associated pathologies (infections, Paget Syndrome, etc.) and a surgery wait of $>48$ hours.

The 209 patients were then divided into three groups, for the treatment with different cephalo-medullary nails.

First group, straight nail (Gamma3 (C) Stryker Mahwah USA) and a cephalic screw. 72 patients of which 19 males and 54 females between the ages of 59 and 97 (average age 83.58), 71 patients had fractures $\mathrm{AO}$ classification types 31.A1-A2 and only one case was type A3. According to the classification of the American Society of Anesthesiologist they were 
evaluated as ASA I (10 patients), ASA II (31 patients), ASA III (24 patients) and ASA IV (7 patients).

Second group, straight nail (Affixus (C) Depuy Orthopaedic Inc. Warsaw USA), a cephalic screw and an anti-rotational screw. 68 patients of which 11 males and 57 females between the ages 56 and 102 (average age 85.61), 51 were patients with femoral neck fractures type 31.A1A2 and 17 cases were type A3. According to the classification of the American Society of Anesthesiologist they were evaluated as ASA I (9 patients), ASA II (22 patients), ASA III (32 patients) and ASA IV (5 patients).

Third group, anatomical nail (ZNN (C) Zimmer Natural Nail Warsaw USA) and a cephalic screw. 69 patients of which 9 males and 60 females between the ages of 58 and 94 (average age 80.18), 54 of which were affected by fractures type 31.A1-A2 and 15 cases were type A3. According to the classification of the American Society of Anesthesiologist they were evaluated as ASA I (12 patients), ASA II (13 patients), ASA III (33 patients) and ASA IV (12 patients).

All the patients received an antibiotic prophylaxis pre-operatively and the antithromboembolic therapy with low molecular weight heparin from hospital admission up to 5 weeks after surgery.

The surgical technique was the same for all the patients, with differences related to the characteristics of each nail, a reduction on a traction bed, a cutaneous incision of around 1.5-2 $\mathrm{cm}$ in length, performed by locating the apex of the greater trochanter with an image intensifier and executing the incision $2 \mathrm{~cm}$ proximally to it, the synthesis was then guided with specialized instruments that lock the nail distally.

Regardless of the particular characteristics of each nail, the implants all required correct positioning (via anteroposterior and axial intraoperative fluoroscopic monitoring) of the cephalic screw in the posterior-inferior quadrant of the femoral neck, according to the area scheme by Cleveland ${ }^{[16]}$.

All the patients were placed in a sitting position and started physiotherapy as of the first postoperative day. Full weight on the leg was granted, depending on the individual possibilities, from the $3^{\text {rd }}$ day up to the $20^{\text {th }}$ post-surgical day.

All the patients were followed post-surgically for a total period of 18 months and evaluated using clinical examination (pain, sensation of instability, the limitations of normal activity and movement) and radiographs (anteroposterior and axial) at 1, 2, 6, 12 and 18 months after surgery.

The Harris Hip Score was used, for an objective evaluation of the results, considering the following ranges: 0 to 70: low; 80 to 89: good; 71 to 79: medium; 90 to 100: excellent.

The statistical analysis was performed using the SPSS 20.0 statistical software. The chi-square test was used for the analysis of discrete data and associations between variables. A comparison between averages was performed with the t-test for two independent variables and the ANOVA test for multiple variables. Finally, eventual correlations were verified by means of the Pearson test. 


\section{RESULTS}

Group I: The average operating time was 37 minutes with a minimum of $20^{\prime}$ and a maximum of 70'. The waiting time for the surgery ranged from a minimum of 1 day to a maximum of 5 (average 2.51 days) due to internistic issues or the assumption of anticoagulants with INR alteration. Only in one case was the exposure of the fracture site needed for the purpose of the reduction. Intraoperative acute anaemia occurred in 69 cases, with an average of 1.4 units of blood transfused per patient. The hospitalization time varied from a minimum of 4 days to a maximum of 34 days (average 11.23 days), with 3 episodes of heart failure in patients with cardiac disease and 14 episodes of general post-operative decline.

Group II: The average operating time was 38.82 minutes with a minimum of $20^{\prime}$ and a maximum of $60^{\prime}$. The waiting time for the surgery varied from a minimum of 1 day to a maximum of 5 (average 2.39 days). All the patients needed intraoperative transfusions, with an average of 1.7 units of blood transfused per patient. The exposure of the fracture site in the reduction was necessary in 15 of the cases. The hospital stay ranged from a minimum of 5 days to a maximum of 18 days (average 11.13 days), with one episode of intestinal blockage and worsening of the clinical condition, 1 case of suspected pulmonary embolism and 4 cases of general post-operative decline.

Group III: The average operating time was 34.63 minutes with a minimum of $25^{\prime}$ and a maximum of 55'. The waiting time for the surgery varied from a minimum of 1 day to a maximum of 4 (average 2.18 days). The exposure of the fracture site in the reduction was necessary in 8 cases. Intra-operative transfusions were performed in all the patients with an average of 1.4 units of blood transfused per patient. The hospitalization time varied from a minimum of 4 days to a maximum of 33 days (average 11.62 days). 1 case had renal complications, 1 case had precordial pain (a cardiopathic patient with elevated cardiac enzymes) and 1 case had a urinary infection with a positive blood culture.

\begin{tabular}{|l|l|l|l|l|l|}
\hline & Gamma3 & Affixus & ZNN & Chi-square & p-value \\
\hline $\begin{array}{l}\text { Intraop. Transfusions } \\
\text { (Average N of units) }\end{array}$ & 1.4 & 1.7 & 1.4 & 16.672 & 0.002 \\
\hline $\begin{array}{l}\text { Intraoperative } \\
\text { complications }\end{array}$ & 0 & 0 & 1 & 2.039 & 0.361 \\
\hline $\begin{array}{l}\text { Postoperative } \\
\text { complications }\end{array}$ & 0 & 0 & 1 & 2.039 & 0.361 \\
\hline
\end{tabular}

Table 1: Results of surgery

\begin{tabular}{|l|l|l|l|c|c|}
\hline HHS & Gamma3 & Affixus & ZNN & Chi-square & p-value \\
\hline$<70$ & $13(18, .0 \%)$ & $21(30.8 \%)$ & $14(20.2 \%)$ & 15.871 & 0.014 \\
\hline
\end{tabular}




\begin{tabular}{|l|l|l|l||}
\hline $70-79$ & $15(20.8 \%)$ & $8(11.7 \%)$ & $14(20.2 \%)$ \\
\hline $80-89$ & $33(45.8 \%)$ & $25(36.7 \%)$ & $17(24.6 \%)$ \\
\hline $90-100$ & $11(15.4<\%)$ & $14(20.8 \%)$ & $24(35.0 \%)$ \\
\hline
\end{tabular}

Table 2: HHS results at $6-48$ months

All the patients in the study achieved fracture healing after 6 months.

The Pearson test (Chart 1) showed a poor correlation between the results of the evaluation questions and the variables examined. The only two variables that have been shown to have a statistically significant correlation with the questionnaires were "AGE" and "GENDER", but for both the coefficient is negative and low.

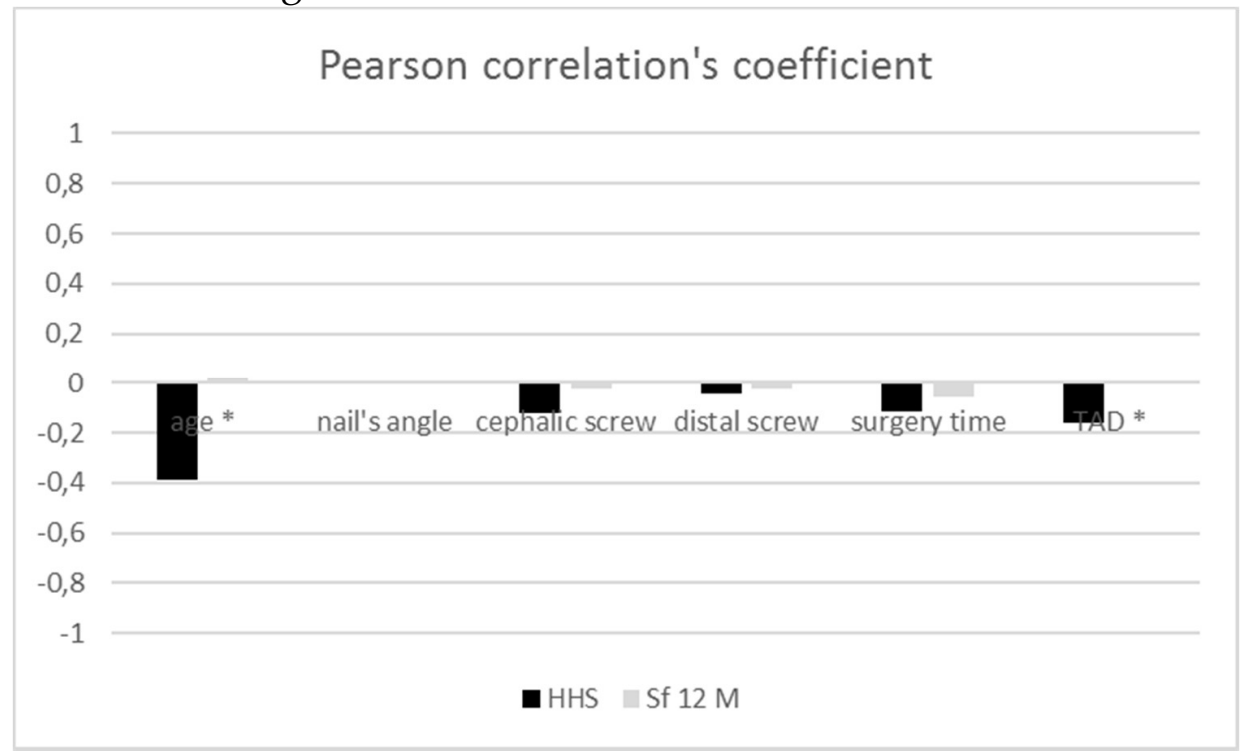

Chart 1. Pearson correlation. P-value $<0,05=*$.

With regard to the association between the variables "TRANSFUSION" and "NAIL" (Chart 2), $51 \%$ of the patients who underwent 1 transfusion belonged to groups I and III; $46 \%$ of those who underwent 2 transfusions belong to Group II. Therefore, a statistically significant difference among the three groups in question has been demonstrated.

Regarding the association between the variables "GENDER" and "SCREW", in the men there was no significant difference between the lengths of the cephalic screws positioned.

In the women, the cephalic screws that are most frequently used have a length of between $85 \mathrm{~mm}$ and $100 \mathrm{~mm}$. 


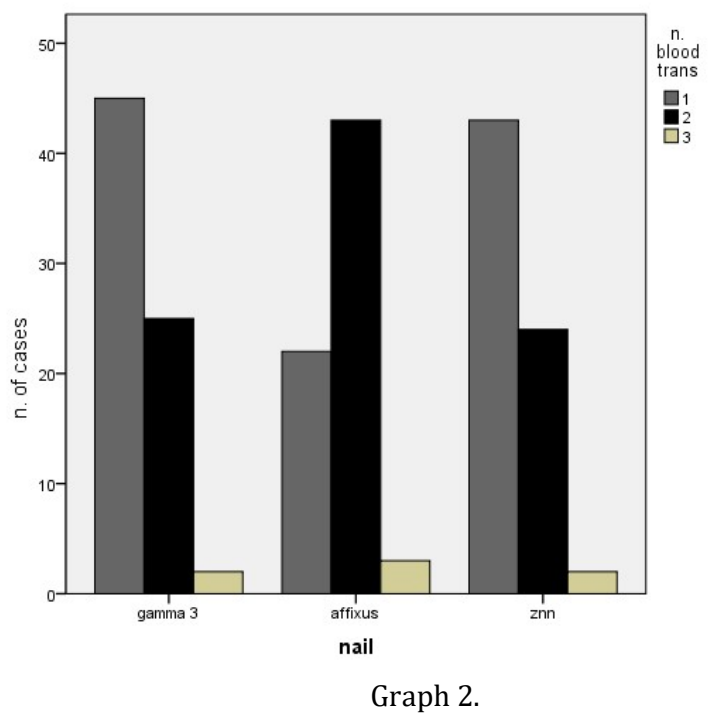

The null hypothesis of the T test (Graph 3) is the following: the average of the cephalic screws (distal) in women is equal to the average of the cephalic screws (distal) in men. It can be stated that, the two averages in question actually differ from each other. In particular, both averages are greater in the men and there is a significant difference between the two genders, both for the length of the cephalic screw ( $p$-value $<0.001$ ) and for the distal one (p-value $<0.001)$. This, in fact, is respectively $103 \mathrm{~mm}$ and $38 \mathrm{~mm}$ in the men and $97 \mathrm{~mm}$ and $34 \mathrm{~mm}$ in the women.

Lastly, analysing with the ANOVA test and the duration of the surgery, both of these variables showed no significant differences among the three groups (p-value 0.154 and 0.805 ).

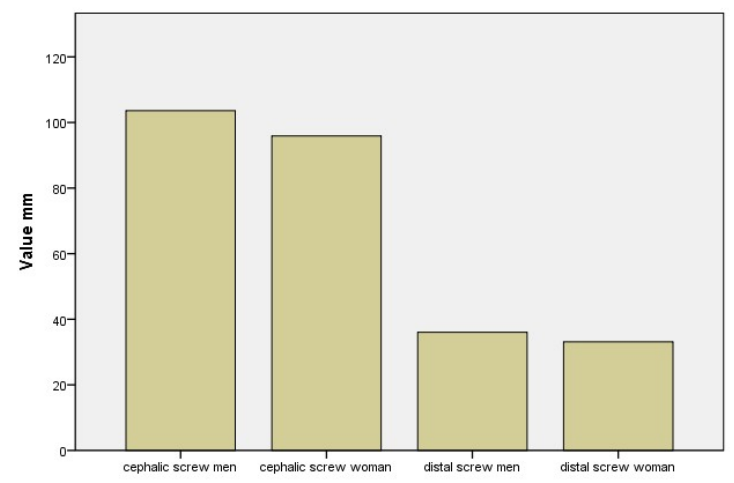

Graph 3. 


\section{DISCUSSION}

The ideal osteosynthesis implant for the treatment of lateral fractures of the neck of the femur is still subject to wide discussion ${ }^{[17]}$. The synthesis with the intramedullary nail (with a miniinvasive technique) is the gold standard treatment for lateral femoral fractures with a biomechanical superiority in relation to the side plates and slide screws ${ }^{[18]}$, less aggressive on the tissue and with a greater respect for the periosteal vascularization, less soft tissue dissection with a decreased operative time, thus preventing excessive blood loss and decreasing the risk of infection, and with an early rehabilitation in order to mobilize and put weight on the leg.

Currently orthopedic surgeons have at their disposal numerous models of trochanteric nails, with different angles of the procurvatum and different dimensions, with 1 or 2 cephalic screws and 1 or 2 distal locking screws, dynamic or static. The innovation of the materials has also led to the use of Titanium, not only for its extreme lightness, but also for the lesser rigidity of the system and the lower coefficient of friction between the individual components.

In literature there are many analyses and meta-analytical studies regarding the different intramedullary nails ${ }^{[5]}$. No study, however, managed to demonstrate a real superiority of one nail over another in relation to the functional outcome in treated patients.

This work has revealed that the intra-operative variables and systemic complications are similar to those described in literature for all three different means of synthesis ${ }^{[19-20]}$.

The rate of serious general complications (cardiac, pulmonary, thromboembolic or cerebrovascular), infections and mortality at 6 months are reported in percentages similar to that of Ekstrom's trial in $2007^{[21]}$ and are obviously independent of the type of nail chosen.

With regard to the cephalic screw, numerous studies have emphasised the correct positioning in order to avoid a cut-out ${ }^{[22]}$. The local complications (cut-out, missed/flawed consolidations and complications in the techniques of fixation), in this study, are absent in accordance with the literature ${ }^{[23-24]}$.

The meta-analysis of the studies that focus the attention on mortality, following a femur fracture, claims that early intervention is the most effective tool to reduce mortality and postoperative complications [25]. Surgery within 48 hours exposes the patient to fewer complications, while surgery after more than 48 hours has more complications, such as pulmonary embolism, DVT, stroke, arrhythmias, pneumonia and sepsis; the incidence of these pathologies increases exponentially with each day that passes from the traumatic episode ${ }^{[26]}$.

From the analysis of our study's results it is clear that, with these 3 different means of synthesis, statistically there are no differences between the time of hospitalization, the duration of the surgery, the bleeding and the functional recovery. These results are in agreement with those reported in literature ${ }^{[27]}$. One statistically significant difference between the 3 means of synthesis analysed is the number of transfusions carried out, which were numerically lower for the Gamma3 $\odot$ and ZNN $\odot$ nails. This is explained by the fact that the 
patients operated with the Affixus (C) nail had a higher percentage of "open" reductions (with exposure of the fracture site).

\section{CONCLUSIONS}

The data analysed in this study is based on a homogeneous sample (patient age, type of fracture and single operator), with a follow-up of 18 months. The limits of this study are related to the fact that it is a retrospective study and at the advanced age of the group of patients examined prevents a long-term evaluation.

The Gamma (c), Affixus (c) and ZNN ( $)$ nails have all provided a stable synthesis, with clinical and radiological evidence of healing. There were no advantages in the use of one intramedullary nail over another and, in particular, by observing the complications and patient outcome, there were no statistically significant differences highlighted.

Therefore, the reduction and intramedullary synthesis performed with the a correct, rigorous and repeatable technique allows the obtainment of a good clinical, radiographic and functional outcome, regardless of the intramedullary nail chosen. The surgical knowledge of the individual operator is, in fact, the determining factor for the treatment of lateral fractures of the femoral neck.

\section{BIBLIOGRAPHY}

1. Osteosynthesis systems in fragility fracture. Sessa G, Evola FR, Costarella L. Aging Clin Exp Res. 2011 Apr;23(2 Suppl):69-70.

2. International Osteoporosis Foundation Osteoporosis in the European Community: a call for action. IOF 2001.

3. Orthopaedic Trauma Association Committee for Coding and Classification. Fracture and dislocation compendium. J Orthop Trauma 1996; 10 (suppl. 1):31-2.

4. Intramedullary versus extramedullary fixation for the treatment of intertrochanteric hip fractures. Baumgaertner MR, Curtin SL, Lindskog DM. Clin Orthop Relat Res. 1998 Mar;(348):87-94.

5. Gamma and other cephalocondylic intramedullary nails versus extramedullary implants for extracapsular hip fractures in adults. Parker MJ, Handoll HH. Cochrane Database Syst Rev. 2010 Sep 8;(9):CD000093. doi: 10.1002/14651858.CD000093.pub5.

6. How evolution of the nailing system improves results and reduces orthopedic complications: more than 2000 cases of trochanteric fractures treated with the Gamma Nail System. Pascarella R, Fantasia R, Maresca A, Bettuzzi C, Amendola L, Violini S, Cuoghi F, Sangiovanni P, Cerbasi S, Boriani S, Tigani DS. Musculoskelet Surg. 2016 Apr;100(1):1-8. doi: 10.1007/s12306-015-0391-y. Epub 2015 Dec 14.

7. A study of 44 patients with subtrochanteric fractures treated using long nail and cerclage cables. 
Persiani, Noia, de Cristo, Graci, Gurzi, Villani. Musculoskelet Surg. 2015 Dec;99(3):225-30. doi: 10.1007/s12306-015-0385-9. Epub 2015 Oct 29.

8. The Gamma nail for peritrochanteric fractures. Halder SC. J Bone Joint Surg Br. 1992 May;74(3):3404.

9. The standard Gamma nail: a critical analysis of 1,000 cases. Kukla C1, Heinz T, Gaebler C, Heinze G, Vécsei V. J Trauma. 2001 Jul;51(1):77-83.

10. Preliminary results of the treatment of proximal femoral fractures with the AFFIXUS nail. Kanakaris NK, Noviello C, Saeed Z, Mitrogiannis L, Tosounidis TH, Tartaglia N. Injury. 2015 Nov;46 Suppl 5:S12-7. doi: 10.1016/j.injury.2015.08.007. Epub 2015 Sep 15.

11. Zimmer Natural Nail System Brochure, Zimmer 2010 (8 pp).

12. Zimmer Natural Nail System Cephalomedullary Nail Surgical Technique, Zimmer 2010 (24 pp).

13. Traumatic arthritis of the hip after dislocation and acetabular fractures: treatment by mold arthroplasty. An end-result study using a new method of result evaluation. Harris WH. J Bone Joint Surg Am. 1969 Jun;51(4):737-55.

14. Quality of life after pertrochanteric femoral fractures treated with a $\gamma$ nail: a single center study of 62 patients. Giessauf C, Glehr M, Bernhardt GA, Seibert FJ, Gruber K, Sadoghi P, Leithner A, Gruber G. BMC Musculoskelet Disord. 2012 Oct 30;13:214. doi: 10.1186/1471-2474-13-214.

15. A 12-Item Short-Form Health Survey: construction of scales and preliminary tests of reliability and validity. Ware J Jr, Kosinski M, Keller SD. Med Care. 1996 Mar;34(3):220-33.

16. A ten-year analysis of intertrochanteric fractures of the femur.Cleveland M, Bosworth DM, Thompson FR, Wilson HJ Jr, Ishizuka T. J Bone Joint Surg Am. 1959 Dec;41-A:1399-408.

17. Systematic effects of surgical treatment of hip fractures: gliding screw-plating vs intramedullary nailing. Verettas DA, Ifantidis P, Chatzipapas CN, Drosos GI, Xarchas KC, Chloropoulou P, Kazakos KI, Trypsianis G, Ververidis A. Injury. 2010 Mar;41(3):279-84. doi: 10.1016/j.injury.2009.09.012.

18. Which Fixation Device is Preferred for Surgical Treatment of Intertrochanteric Hip Fractures in the United States? A Survey of Orthopaedic Surgeons. Niu E, Yang A, Harris AH, Bishop J. Clin Orthop Relat Res. 2015 Nov;473(11):3647-55. doi: 10.1007/s11999-015-4469-5. Epub 2015 Jul 25.

19. IMHS clinical experience in the treatment of peritrochanteric fractures. The results of a multicentric Italian study of 981 cases. Rebuzzi E, Pannone A, Schiavetti S, Santoriello P, de Nicola U, Fancellu G, Cau P, Gulli S, Dordolin P, Maniscalco P, Morici F, Commessatti M, Pozzi-Mucelli M, Maiorana CS, Bassini F. Injury. 2002 Jun;33(5):407-12.

20. A randomized study of the compression hip screw and Gamma nail in 426 fractures. Ahrengart L, Törnkvist H, Fornander P, Thorngren KG, Pasanen L, Wahlström P, Honkonen S, Lindgren U. Clin Orthop Relat Res. 2002 Aug;(401):209-22.

21. Functional outcome in treatment of unstable trochanteric and subtrochanteric fractures with the proximal femoral nail and the Medoff sliding plate. Ekström W, Karlsson-Thur C, Larsson S, Ragnarsson B, Alberts KA. J Orthop Trauma. 2007 Jan;21(1):18-25.

22. A laboratory model to evaluate cutout resistance of implants for pertrochanteric fracture fixation. Sommers MB, Roth C, Hall H, Kam BC, Ehmke LW, Krieg JC, Madey SM, Bottlang M. J Orthop Trauma. 2004 Jul;18(6):361-8. 
23. The standard Gamma nail or the Medoff sliding plate for unstable trochanteric and subtrochanteric fractures. A randomised, controlled trial. Miedel R, Ponzer S, Törnkvist H, Söderqvist A, Tidermark J. J Bone Joint Surg Br. 2005 Jan;87(1):68-75.

24. Comparison of clinical outcomes with three different intramedullary nailing devices in the treatment of unstable trochanteric fractures. Zehir S1, Şahin E2, Zehir R3. Ulus Travma Acil Cerrahi Derg. 2015 Nov;21(6):469-76. doi: 10.5505/tites.2015.28227.

25. Length of stay, mortality, morbidity and delay to surgery in hip fractures. Lefaivre KA, Macadam SA, Davidson DJ, Gandhi R, Chan H, Broekhuyse HM. J Bone Joint Surg Br. 2009 Jul;91(7):922-7. doi: 10.1302/0301-620X.91B7.22446.

26. Does timing of surgery matter in fragility hip fractures? Leung F, Lau TW, Kwan K, Chow SP, Kung AW. Osteoporos Int. 2010 Dec;21(Suppl 4):S529-34. doi: 10.1007/s00198-010-13912. Epub 2010 Nov 6.

27. Use of the Gamma3 ${ }^{\mathrm{TM}}$ nail in a teaching hospital for trochanteric fractures: mechanical complications, functional outcomes, and quality of life. Buecking B, Bliemel C, Struewer J, Eschbach D, Ruchholtz S, Müller T. BMC Res Notes. 2012 Nov 23;5:651. doi: 10.1186/17560500-5-651. 